\title{
GAPS BETWEEN FRACTIONAL PARTS, AND ADDITIVE COMBINATORICS
}

\author{
Antal Balog, Andrew Granville and Jozsef Solymosi \\ Abstract. We give bounds on the number of distinct differences $N_{a}-a$ as $a$ varies over all \\ elements of a given finite set $A$, and $N_{a}$ is a nearest neighbour to $a$.
}

\section{INTRODUCTION}

Let $\alpha$ be a real, irrational number and consider the set of points

$$
S_{\alpha}(N)=\{\alpha n: 1 \leq n \leq N\} \subseteq \mathbb{R} / \mathbb{Z},
$$

which is isomorphic to the set of points $\left\{e^{2 i \pi \alpha n}: 1 \leq n \leq N\right\}$ on the unit circle $S^{1}$. Moreover, one can view $S_{\alpha}(N)$ as the set of points $\{\{\alpha n\}: n \leq N\} \subseteq[0,1)$, where $\{t\}$ denotes the fractional part of real number $t$ (that is, $\{t\}=t-[t]$ where $[t]$ is the largest integer $\leq t$ ), and then order them as

$$
S_{\alpha}(N)=\left\{0 \leq b_{1}<b_{2}<\ldots<b_{N}<1\right\} .
$$

In 1957 Steinhaus observed, and in 1958 Vera Sós proved, $[7,8]^{1}$, that there are, at most, three distinct consecutive differences in $S_{\alpha}(N)$, that is

$$
\left|\left\{b_{i+1}-b_{i}: 1 \leq i \leq N\right\}\right| \leq 3
$$

where we take $b_{N+1}=1+b_{1}$. This is arguably surprising since it is well known that $S_{\alpha}(N)$ becomes increasingly uniformly distributed $\bmod 1$ as $N$ gets larger, that is $S_{\alpha}(N)$ looks random globally for large $N$, whereas Sós's result tells us that $S_{\alpha}(N)$ looks highly structured locally. In 2002, Vâjâitu and Zaharescu [10] considered a question in-between these two extremes: Take the set of $b_{i}$ 's above and erase as many elements of the set as one likes - how large can one make the resulting set of differences? They proved the following result (though with the constant $2+2 \sqrt{2}$ in place of our $2 \sqrt{2}$ ):

The first author is partially support by Hungarian National Research Grant K 109789, the second author is partially supported by NSERC, and the third author is partially supported by ERC Advanced Research Grant no 267165 (DISCONV), by Hungarian National Research Grant NK 104183, and by NSERC.

${ }^{1}$ As well as Surányi, Swierczkowski [9], Szüsz, and Erdős and Turán 
Corollary 2. For any subset $A$ of $S_{\alpha}(N)$ there are no more than $2 \sqrt{2 N}+1$ distinct consecutive differences in $A$; that is, if $A=\left\{0 \leq a_{1}<a_{2}<\ldots<a_{m}<1 \leq a_{m+1}:=\right.$ $\left.1+a_{1}\right\} \subset S_{\alpha}(N)$ then

$$
\left|\left\{a_{j+1}-a_{j}: 1 \leq j \leq m\right\}\right| \leq 2 \sqrt{2 N}+1 .
$$

Moreover there exists $A \subseteq S_{\alpha}(N)$ with at least $\sqrt{2 N}-1$ distinct consecutive differences, so the result here is "best possible" up to the value of the constant in front of the " $\sqrt{N}$ ".

The proof in [10] comes from careful number theoretic considerations, whereas our proof stems from more general additive combinatorics ideas, emerging from the fact that $S_{\alpha}(N)+S_{\alpha}(N)$ is not much larger than $S_{\alpha}(N)$. Here and throughout we define

$$
A+B:=\{n: n=a+b \text { for some } a \in A, b \in B\} .
$$

A set of points in $\mathbb{R} / \mathbb{Z}$ has a natural "circular" ordering by starting at any given point and then proceeding anticlockwise. Evidently there is no generic place to start the ordering but this will not effect on our results. Each of the points will have a smaller and a larger neighbour, the two points that appear before and after our given point in the ordering, respectively (where, for the purposes of this definition, the first point in our ordering appears after the last).

We need a notion of length in $\mathbb{R} / \mathbb{Z}$ : Any $t \in \mathbb{R} / \mathbb{Z}$ represents the coset $t+\mathbb{Z}$, and we define $\|t\|$ to be the minimal size of any element of $t+\mathbb{Z}$, that is

$$
\|t\|:=\min _{n \in \mathbb{Z}}|t-n| \text {. }
$$

Hence the distance between two points in $x, y \in \mathbb{R} / \mathbb{Z}$ is given by $\|x-y\|$.

Theorem 1. If $B$ is a finite subset of $\mathbb{R} / \mathbb{Z}$ then any subset $A$ of $B$ has at most

$$
\sqrt{2|B|} \frac{|A+B|}{|B|}+1
$$

distinct, consecutive differences. Moreover there are subsets $A$ of $B$ with at least $\sqrt{2|B|}-1$ distinct consecutive differences.

The bound in Theorem 1 is better than the trivial bound, $\leq|A|$, if $|A+B| \ll|A| \sqrt{|B|}$.

A map $\phi: A \rightarrow B$ is called a 2-isomorphism if $a_{1}-a_{2}=a_{3}-a_{4}$ if and only if $\phi\left(a_{1}\right)-\phi\left(a_{2}\right)=\phi\left(a_{3}\right)-\phi\left(a_{4}\right)$ for all $a_{1}, a_{2}, a_{3}, a_{4}$. Note that this does not effect coincidences amongst the set of differences of elements of $A$. Any non-degenerate affine transformation is a 2-isomorphism on the reals, so that any given set of reals is 2 -isomorphic to a subset of $\left[0, \frac{1}{2}\right)$. The natural embedding $\left[0, \frac{1}{2}\right) \rightarrow \mathbb{R} / \mathbb{Z}$ given by sending $t$ to $t+\mathbb{Z}$ is a 2 -isomorphism, and the composition of these two 2-isomorphisms is also a 2-isomorphism. Hence we can apply Theorem 1 to any finite set of reals.

For any ordered set $b_{1}<b_{2}<\ldots<b_{m}$ define

$$
D(B):=\left\{b_{i+1}-b_{i}: 1 \leq i \leq m-1\right\}
$$

to be the set of distinct consecutive differences between elements of $B$. Since $A+B \subseteq B+B$, we immediately deduce the following result from Theorem 1: 
Corollary 1. If $B$ is a finite set of real numbers then

$$
\sqrt{2|B|}-1 \leq \max _{A \subseteq B}|D(A)| \leq \sqrt{2|B|} \cdot \frac{|B+B|}{|B|}+1 .
$$

A nice example is given by the projection of the lattice points of a compact subset of a $k$-dimensional lattice onto $\mathbb{R} / \mathbb{Z}$. That is for given real numbers $\alpha_{1}, \ldots, \alpha_{n}$ and integers $N_{1}, \ldots, N_{k} \geq 1$, the set

$$
B=\left\{n_{1} \alpha_{1}+n_{2} \alpha_{2}+\ldots+n_{k} \alpha_{k}: 0 \leq n_{j}<N_{j} \text { for } 1 \leq j \leq k\right\} \subset \mathbb{R} / \mathbb{Z}
$$

typically satisfies $|B+B| \leq 2^{k}|B|$, so the upper and lower bounds in Corollary 1 are equal up to a factor $\lesssim 2^{k}$. Cobeli et al [3], discovered and proved the following, wonderful generalization of Steinhaus's problem: If the elements of $B$ are $0 \leq b_{1}<b_{2}<\ldots<b_{N}<1$ then there exists a set $\left\{r_{1}, \ldots, r_{l}\right\}$ of positive real numbers with $l \leq 2^{k}$, such that every difference $b_{j}-b_{i}$ with $1 \leq i<j \leq N$ can be written as a sum of the $r_{i}$ 's. Rather more generally:

Theorem 2. Let $B$ be a finite subset of $\mathbb{R} / \mathbb{Z}$, and $C$ be any subset of $B$ for which $C-$ $B=B-B$. Let $b_{c}^{-}$and $b_{c}^{+}$be the smaller and larger neighbours of $c$ in $B$, and let $R^{-}:=\left\{c-b_{c}^{-}: c \in C\right\}, R^{+}:=\left\{c-b_{c}^{+}: c \in C\right\}$. Then every element of $B-B$ is a sum of elements of $R^{-}$, as well as a sum of elements of $R^{+}$, that is $B-B \subset\left\langle R^{-}\right\rangle_{\mathbb{N}_{0}}=\left\langle R^{+}\right\rangle_{\mathbb{N}_{0}}$. $\left(\right.$ Here $\mathbb{N}_{0}=\mathbb{N} \cup\{0\}$.)

For $B$ as in (2) we can take

$$
C=\left\{\delta_{1} N_{1} \alpha_{1}+\delta_{2} N_{2} \alpha_{2}+\ldots+\delta_{k} N_{k} \alpha_{k}: \text { each } \delta_{i}=0 \text { or } 1\right\}
$$

so that $\left|R^{-}\right|,\left|R^{+}\right| \leq|C| \leq 2^{k}$, which is comparable to $|B+B| /|B|$.

However, we have been unable, in general, to estimate the size of $C$ in terms of quantities associated with $B$, and there does not seem to be a close link between $|C|$ and $|B+B| /|B|$ in general. Let $r_{3}(N)$ denote the size of the largest set $S \subset\{1, \ldots, N\}$ containing no non-trivial 3-term arithmetic progressions. As is well-known, $r_{3}(N) \gg N e^{-\delta \sqrt{\log N}}$ for some constant $\delta>0$, thanks to a clever construction of Behrend [1].

Proposition 1. For any given integer $N$ there exists a finite set of $N$ integers $B$ for which $|B+B| \leq 10 N$ and if $C \subset B, C-B=B-B$ then $|C| \geq r_{3}(N)$.

The length of $t \in(\mathbb{R} / \mathbb{Z})^{d}$ is defined to be the minimal length of any element of $t+\mathbb{Z}^{d}$; that is

$$
\|t\|:=\min _{n \in \mathbb{Z}^{d}}|t-n|=\left(\sum_{i=1}^{d} \min _{n \in \mathbb{Z}}\left|t_{i}-n_{i}\right|^{2}\right)^{1 / 2}
$$

where $t=\left(t_{1}, \ldots, t_{d}\right)$. The distance between $x, y \in(\mathbb{R} / \mathbb{Z})^{d}$ is then given by $\|x-y\|$.

For a given finite subset $A$ of $(\mathbb{R} / \mathbb{Z})^{d}$ and $a \in A$, let $N_{a}$ be one of the elements of $A$ that is nearest to $a$ in the $\|$.$\| -norm.. We now investigate the number of possible vectors$ $N_{a}-a$ as we vary over $a \in A$. 
Theorem 3. Let $w_{n}=\left(\alpha_{1} n, \alpha_{2} n, \ldots, \alpha_{d} n\right) \in(\mathbb{R} / \mathbb{Z})^{d}$. For each $i \in[1, N]$ select $j=$ $j(i) \in[1, N]$ with $j \neq i$ so that $\left\|w_{i}-w_{j}\right\|$ is minimized. There are $\ll(4 / 3)^{d}$ distinct elements of the set $D=\left\{ \pm\left(w_{i}-w_{j(i)}\right): i=1,2 \ldots, N\right\}$.

Unfortunately for a general set $A \in(\mathbb{R} / \mathbb{Z})^{d}$ we can say somewhat less, namely we can prove a similar bound only for a larger subset of $A$.

Theorem 4. Let $A$ be a finite subset of $(\mathbb{R} / \mathbb{Z})^{d}$, and $\epsilon>0$ be a small real number. There exists $A^{\prime} \subset A$, with $\geq(1-\epsilon)|A|$ elements, such that

$$
\left|\left\{N_{a}-a: a \in A^{\prime}\right\}\right| \ll(4 / 3)^{d} \cdot \min _{B} \frac{|A+B|^{2}}{|A||B|} \cdot \frac{1}{\epsilon} \log \frac{1}{\epsilon} .
$$

We give an example in section 5 to show that this cannot be much improved.

It would be interesting to determine $\min _{B} \frac{|A+B|^{2}}{|A||B|}$ for an arbitrary set $A$ of real numbers. Taking $B=\{b\}$, any one element set, or taking $B=A$, evidently shows

$$
\min _{B} \frac{|A+B|^{2}}{|A||B|} \leq \min \left\{|A|,(|A+A| /|A|)^{2}\right\}
$$

\section{The Three GAPs TheOREM AND BEYOND}

We begin with recalling Liang's elegant solution [6] to Steinhaus's problem. We do so not only because it inspires some of our arguments, but also because we want to demonstrate how simple and short it is.

The Three Gaps theorem. Reorder $S_{\alpha}(N)$ as $0 \leq\left\{a_{1} \alpha\right\}<\left\{a_{2} \alpha\right\}<\ldots<\left\{a_{N} \alpha\right\}<1$. Then the consecutive differences $\left\{a_{i+1} \alpha\right\}-\left\{a_{i} \alpha\right\}, 1 \leq i \leq N-1$ each equal one of three numbers, namely the distances between each pair of points amongst $\left\{a_{N} \alpha\right\}-1,0$ and $\left\{a_{1} \alpha\right\}$.

Proof sketch. $D(A)$ is contained in the set $G$ of gaps $\left\{a_{i+1} \alpha\right\}-\left\{a_{i} \alpha\right\}$ for which $\left\{\left(a_{i}-1\right) \alpha\right\}$ and $\left\{\left(a_{i+1}-1\right) \alpha\right\}$ are not consecutive elements of the re-organized sequence (that is, we select one representative from each set of gaps that are obviously the same length.) Note that if $\left\{\left(a_{i}-1\right) \alpha\right\}$ and $\left\{\left(a_{i+1}-1\right) \alpha\right\}$ are not consecutive elements of the re-organized sequence then either $a_{i}=1$ or $a_{i+1}=1$, or there is some $\left\{a_{j} \alpha\right\} \in\left(\left\{\left(a_{i}-1\right) \alpha\right\},\left\{\left(a_{i+1}-\right.\right.\right.$ 1) $\alpha\})$. This last possibility implies that $\left\{\left(a_{j}+1\right) \alpha\right\} \in\left(\left\{a_{i} \alpha\right\},\left\{a_{i+1} \alpha\right\}\right)$, and so $a_{j}=N$. Hence $|G| \leq 3$ and so there are at most three distinct gaps. When $a_{i}=1$ the gap is the distance between 0 and $\left.\left\{\left(a_{i+1}-1\right) \alpha\right\}\right)$; when $a_{i+1}=1$ the gap is the distance between $\left\{\left(a_{i}-1\right) \alpha\right\}$ and 0 ; and when $\left.\left\{a_{i} \alpha\right\}<\{(N+1) \alpha\}<\left\{a_{i+1} \alpha\right\}\right)$ the gap is the sum of the two previous distances.

Example. The Weierstrass parametrization $\mathbb{C} / \Lambda \rightarrow E$ of an elliptic curve $E$, is an isomorphism sending $z \in \mathbb{C}$ to $P_{z}:=\left(\wp(z), \wp^{\prime}(z)\right)$, for a certain function $\wp$ and 2-dimensional lattice $\Lambda$. Hence $P_{m z}=m P_{z}$. The multiples $P, 2 P, \ldots, N P$ of a given point $P$ on the real locus of $E$ (for example, the point $P=(1,3)$ on $E: y^{2}=x^{3}+8 x$ ) all lie on the real 
locus of $E$, which is one continuous curve. Moving along this curve from $-\infty$ to $+\infty$, we encounter these points in some order $a_{1} P, a_{2} P, \ldots, a_{N} P$. As noted in [5], the Three Gaps theorem implies that there are, at most, three distinct differences $\left(a_{i+1}-a_{i}\right) P$.

Chung and Graham [2] came up with a beautiful generalization of the Three Gaps theorem, and Liang's proof works here too.

The $3 k$-Gaps theorem. Let $A$ be the set of elements $\beta_{i}+n_{i} \alpha \in \mathbb{R} / \mathbb{Z}$ for $1 \leq n_{i} \leq N_{i}$ and $i=1,2, \ldots, k$. Then the sizes of consecutive differences of $A$ have $\leq 3 k$ different values.

Proof. List the elements of $A$ in an anticlockwise "circular order", $a_{1}, a_{2}, \ldots, a_{N}$, where each $a_{j}$ is in the form $\beta+n \alpha$ with some $\beta=\beta_{i}$ and $n=n_{i}$. Note that $N=N_{1}+\cdots+N_{k}$, and $D(A)$, the set of consecutive differences is $\left\{a_{2}-a_{1}, a_{3}-a_{2}, \ldots, a_{N}-a_{N-1}, a_{1}-a_{N}\right\}$. We show that $|D(A)| \leq 3 k . D(A)$ is contained in the set of gaps (better to call them arcs) $a_{i+1}-a_{i}$ for which $a_{i}+\alpha$ and $a_{i+1}+\alpha$ are not consecutive elements of $A$. This can only happen if either one of these two points (or both) is not an element of $A$, or there is an element $a \in A$ between them, but $a-\alpha$ is not an element of $A$. That is either one of the endpoints of the $A$-free $\operatorname{arc}\left(a_{i}, a_{i+1}\right)$ is of the form $\beta_{j}+N_{j} \alpha$ or there is a point of the form $\beta_{j}$ on the $A$-free $\operatorname{arc}\left(a_{i}, a_{i+1}\right)$. That is no more then 3 options for each $j$.

\section{Bounding the number of Differences in $A$}

Proof of the first part of Theorem 1. Order the elements of $A$ as $a_{1}, a_{2}, \ldots, a_{m}$, going anticlockwise around the circle. For each $d \in D(A)$ select $i(d)$ for which $a_{i(d)+1}-a_{i(d)}=d$ and let

$$
J_{A}:=\{i(d): d \in D(A)\}
$$

For a $k$ chosen optimally later, we partition the set $A+B$ into $k \operatorname{arcs} I_{1}, I_{2}, \ldots, I_{k}$ each containing roughly the same number of elements of $A+B$. We count the number, $P$, of pairs $(i, b)$ with $i \in J_{A}$ and $b \in B$ for which $a_{i}+b$ and $a_{i+1}+b$ both lie in the same arc $I_{j}$. For each fixed $b$, the points of $A+b$ follow each other in the order

$$
a_{1}+b, a_{2}+b, \ldots, a_{m}+b
$$

and so consecutive numbers here lie in the same arc unless they straddle the boundary between consecutive arcs, which can happen for at most $k$ pairs. Therefore

$$
P \geq|B|(|D(A)|-k)
$$

On the other hand, if we are given a pair of integers $u$ and $v$ which must equal to $a_{i}+b$ and $a_{i+1}+b$ with some $i \in J_{A}$ and $b \in B$, then their difference is $v-u=d \in D(A)$, so $i=i(d)$ where $d=a_{i+1}-a_{i}$ which are thus uniquely determined and hence so is $b=u-a_{i}\left(=v-a_{i+1}\right)$, therefore there is, at most, one such pair $(i, b)$. We therefore deduce that

$$
P \leq \sum_{j=1}^{k}\left(\begin{array}{c}
\left|I_{j}\right| \\
2
\end{array}\right)
$$


where $\left|I_{j}\right|$ denotes the number of elements of $A+B$ in $I_{j}$. If $|A+B|=k L+r, 0 \leq r<k$, then one can certainly arrange the end points of the $\operatorname{arcs} I_{j}$ so that $r$ of the $\left|I_{j}\right|$ are $=L+1$, the other $k-r$ of the $\left|I_{j}\right|$ are $=L$, in which case

$$
P \leq r\left(\begin{array}{c}
L+1 \\
2
\end{array}\right)+(k-r)\left(\begin{array}{l}
L \\
2
\end{array}\right)=\frac{(k L+r)^{2}}{2 k}-\frac{r^{2}}{2 k}-\frac{k L}{2} \leq \frac{|A+B|^{2}}{2 k}
$$

Comparing this with (3) gives the upper bound

$$
|D(A)| \leq k+\frac{|A+B|^{2}}{2 k|B|}
$$

which we minimize by selecting $k$ to be the integer satisfying $\frac{|A+B|}{\sqrt{2|B|}}+1>k \geq \frac{|A+B|}{\sqrt{2|B|}}$. This implies the result.

Deduction of Corollary 2. $S_{\alpha}(N)=\{\alpha n: 1 \leq n \leq N\}$ is an arithmetic progression, and $S_{\alpha}(N)+S_{\alpha}(N)=S_{\alpha}(2 N) \backslash\{\alpha\}$, so we have $\left|S_{\alpha}(N)+S_{\alpha}(N)\right|=2 N-1$. The result readily follows from Corollary 1 (or taking $A=B=S_{\alpha}(N)$ in Theorem 1).

Proof of the second part of Theorem 1. (Construction of $A$ with a lot of differences by the greedy algorithm.) Let $a_{1}=b_{1}, a_{2}=b_{2}$. We select each $a_{j}=b_{k_{j}}, j \geq 3$ to be the minimum $k>k_{j-1}$ such that $b_{k}-a_{j-1}$ is different from the previous consecutive differences, $a_{2}-a_{1}, a_{3}-a_{2}, \ldots, a_{j-1}-a_{j-2}$. Evidently we need to avoid $j-2$ values so $k_{j} \leq k_{j-1}+j-1$. Hence

$$
k_{j} \leq(j-1)+(j-2)+\ldots+1+1=\left(\begin{array}{l}
j \\
2
\end{array}\right)+1
$$

and there is room for choosing $k_{j}$ while this is $\leq|B|$. Hence we can take $\sqrt{2|B|}>j \geq$ $\sqrt{2|B|}-1$. This gives the second part of Theorem 1 .

Another proof, though with a weaker constant, follows from recalling that every set $B$ contains a Sidon set $A$ of size $\gg \sqrt{|B|}$; and that there are $|A|-1$ distinct differences between consecutive elements of a Sidon set $A$.

\section{The number of DifFEREnCES In the ORIGINAL SET}

Proof of Theorem 2. Think about our sets in the anticlockwise "circular order". For any $b^{\prime}, b^{\prime \prime} \in B$ there exists $c \in C, b \in B$ such that $c-b=b^{\prime}-b^{\prime \prime}$. Consider the anti-clockwise oriented arc from $b$ to $c$, this has the same arc length as the anti-clockwise oriented arc from $b^{\prime \prime}$ to $b^{\prime}$. Locate the points $b=b_{0}, \ldots, b_{r}=c$ of $B$ sitting on this arc and ordered in anti-clockwise direction. Each $b_{i+1}-b_{i}$ is of the form $c^{*}-b^{*}$, and we can keep dividing those arcs into smaller and smaller parts until they can get no smaller. But then the parts must be of the form $c-b_{c}^{-}$where there are no elements in-between $c$ and $b_{c}^{-}$. Hence, $b_{c}^{-}$must be the smaller neighbour of $c$. This proves the first half of the theorem. The 
other half comes from the exact same analysis with choosing clockwise oriented arcs at the very beginning and always after. Finally, to see that $\left\langle R^{-}\right\rangle_{\mathbb{N}_{0}}=\left\langle R^{+}\right\rangle_{\mathbb{N}_{0}}$ note that both $R^{-} \subset B-B \subset\left\langle R^{+}\right\rangle_{\mathbb{N}_{0}}$ and $R^{+} \subset B-B \subset\left\langle R^{-}\right\rangle_{\mathbb{N}_{0}}$.

Proof of Proposition 1. Let $S$ be a set of positive integers $\leq N$, which contains no nontrivial 3-term arithmetic progressions. That is, if $2 s=s_{1}+s_{2}$ in $S$, then $s_{1}=s_{2}=s$. There is such a set of $r_{3}(N)$ elements. Select $x=5 N-2|S|$. Let

$$
B=S \cup\{2 N<n \leq x-2 N\} \cup(x-S),
$$

so that $|B|=x-4 N+2|S|=N$ and $B+B \subseteq\{1,2, \ldots, 2 x\}$, so that $|B+B| \leq 2 x<10 N$. Now for each $s \in S$ consider $m=x-2 s=(x-s)-s \in B-B$. Note that $m \geq x-2 N$. If for some $b_{1}, b_{2} \in B$ we have $m=b_{1}-b_{2}$ then $b_{1}>x-2 N$, else $b_{2}=b_{1}-m \leq$ $x-2 N-(x-2 N)$ which is impossible, so $b_{1}=x-s_{1}, s_{1} \in S$. Similarly $b_{2} \leq 2 N$ else $b_{1}=b_{2}+m>2 N+(x-2 N)$ which is impossible, and so $b_{2}=s_{2}, s_{2} \in S$. Therefore $2 s=x-m=x-b_{1}+b_{2}=s_{1}+s_{2}$, which is only possible in $S$ when $s_{1}=s_{2}=s$. Hence if $C-B=B-B$ then $x-S \subset C$, and so $|C| \geq|S|=r_{3}(N)$.

\section{NEAREST NEIGHBOURS}

In this section we investigate the set $\left\{N_{a}-a: a \in A\right\}$ of vectors from points of $A$ to their nearest neighbours $N_{a}$ in $A$.

We begin with an important lemma from the theory of sphere packing:

Lemma 1. There exists an absolute constant $\kappa>0$ such that if $z_{1}, z_{2}, \ldots, z_{k} \in \mathbb{R}^{d}$ with $\left|z_{i}\right|=1$ and $\left|z_{i}-z_{j}\right| \geq 1$ for all $i \neq j$ then $k \leq \kappa(4 / 3)^{d}$.

This is the celebrated "kissing problem": how many points can one place on the surface of a unit sphere such that they are all separated by Euclidean distance at least 1? The exact answer is known only for $d=1,2,3,4,8$, and 24. According to [4, pages 23-24], the best bounds known in general are $1.15^{d} \ll k \leq \kappa \cdot 1.33^{d}$.

Lemma 2. There exists an absolute constant $\kappa>0$ such that if $z_{1}, z_{2}, \ldots, z_{k} \in(\mathbb{R} / \mathbb{Z})^{d}$ with $\left\|z_{i}-z_{j}\right\| \geq \max \left\{\left\|z_{i}\right\|,\left\|z_{j}\right\|\right\}$ for all $i \neq j$ then $k \leq \kappa(4 / 3)^{d}$.

Proof. For each $z_{i} \in(\mathbb{R} / \mathbb{Z})^{d}$ we choose that representative, $\tilde{z}_{i} \in \mathbb{R}^{d}$ which is closest to the origin. Draw a "radial half line" from the origin across each $\tilde{z}_{i}$. Note that $\left\|z_{i}\right\|$ is precisely the Euclidean length of $\tilde{z_{i}}$. Moreover, $\left|\tilde{z_{i}}-\tilde{z_{j}}\right| \geq|| z_{i}-z_{j} \|$, because this latter one is the minimal distance between any representatives of the two points. Thus we have

$$
\left|\tilde{z_{i}}-\tilde{z_{j}}\right| \geq \max \left\{\left|\tilde{z}_{i}\right|,\left|\tilde{z}_{j}\right|\right\} \text { for all } i \neq j \text {. }
$$

Now we have $k$ half lines, starting from the origin and each supporting exactly one point $\tilde{z}_{i}$. Two points on one half line violates (4). Even more, any two such radial lines must be at an angle of at least $\pi / 3$, a smaller angle also violates (4). If $r=\max _{j}\left|\tilde{z_{j}}\right|$ then we can move each point out to the circumference of the sphere of radius $r$ along its "radial half line". This spreads the points out as far as possible since the angle between any two radial lines is at least $\pi / 3$. A renormalized Lemma 2 proves the statement. 
Corollary 3. There exists an absolute constant $\kappa>0$ such that any $z \in(\mathbb{R} / \mathbb{Z})^{d}$ belongs to $\leq \kappa(4 / 3)^{d}$ balls of the form $B_{a}\left(\left\|N_{a}-a\right\|\right)$ (where $B_{x}(r)$ denotes the closed ball of radius $r$ centered at $x$.)

Proof. Suppose that $z \in B_{a_{j}}\left(\left\|N_{a_{j}}-a_{j}\right\|\right)$ for $j=1,2, \ldots, k$. Then $\left\|a_{i}-a_{j}\right\| \geq\left\|N_{a_{j}}-a_{j}\right\| \geq$ $\left\|z-a_{j}\right\|$ whenever $i \neq j$. Writing $a_{j}^{\prime}=z-a_{j}$ for all $j$ we find that $\left\|a_{i}^{\prime}-a_{j}^{\prime}\right\|=\left\|a_{i}-a_{j}\right\| \geq$ $\left\|a_{j}^{\prime}\right\|$ for all $i \neq j$, and so $k<\kappa(4 / 3)^{d}$ by Lemma 2 .

Proof of Theorem 3. Note that $w_{i}-w_{j}=w_{i-j} \in(\mathbb{R} / \mathbb{Z})^{d}$, and $w_{-i}=-w_{i}$, hence $D$ is a subset of $\left\{ \pm w_{1}, \pm w_{2}, \ldots, \pm w_{N-1}\right\}$, which we re-order so that $\left\|w_{k_{1}}\right\| \leq\left\|w_{k_{2}}\right\| \leq \ldots$ Recall that $j(i)$ minimizes $\left\|w_{i}-w_{j(i)}\right\|$. If $j=i+k$ or $j=i-k$ then $\left\|w_{i}-w_{j}\right\|=\left\|w_{k}\right\|$, so to find $j(i)$ we have to find the smallest integer $\ell$ such that either $i+k_{\ell}$ or $i-k_{\ell}$ falls into the interval $[1, N]$. Observe that if $k \leq \frac{N}{2}$ then $i+k$ is a legal choice for all $i \leq \frac{N}{2}$, and $i-k$ is for all $i>\frac{N}{2}$. Let $\ell$ be the smallest positive integer such that $k_{\ell} \leq \frac{N}{2}$. That means

$k_{1}, \ldots, k_{\ell-1}>\frac{N}{2}$, and as $k_{\ell}$ is a legal choice for every $i$, we have restricted the choices to $D \subset\left\{ \pm w_{k_{1}}, \pm w_{k_{2}}, \ldots, \pm w_{k_{\ell}}\right\}$.

Now if $1 \leq u<v<\ell$ then $\frac{N}{2}<k_{u}, k_{v} \leq N$ so that $j=\left|k_{u}-k_{v}\right|<\frac{N}{2}$ but $k_{\ell}$ is the first element of the reordered sequence which is $\leq \frac{N}{2}$, so $j \geq \ell$. Hence $\left\|w_{k_{u}}-w_{k_{v}}\right\|=\left\|w_{j}\right\| \geq$ $\left\|w_{k_{\ell}}\right\|>\max \left\{\left\|w_{k_{u}}\right\|,\left\|w_{k_{v}}\right\|\right\}$, and the result follows from Lemma 2.

Proof of Theorem 4. Let $B \neq \emptyset$ be any fixed finite subset of $(\mathbb{R} / \mathbb{Z})^{d}$. For any $a \in A$ the ball $B_{a}\left(\left\|N_{a}-a\right\|\right)$ contains no elements of $A$ other than $a$ itself at the center, $N_{a}$ and possibly some others on the boundary. If we translate this ball by an element $b \in B$ then $B_{a+b}\left(\left\|N_{a}-a\right\|\right)$ may contain many elements of $A+B$, but usually it is not the case. To see this, fix an element $b \in B$, and consider

$$
\begin{aligned}
\sum_{a \in A}\left|(A+B) \cap B_{a+b}\left(\left\|N_{a}-a\right\|\right)\right| & =\sum_{c \in A+B} \#\left\{a \in A: c \in B_{a+b}\left(\left\|N_{a}-a\right\|\right)\right\} \\
& =\sum_{c \in A+B} \#\left\{a \in A: c-b \in B_{a}\left(\left\|N_{a}-a\right\|\right)\right\} \leq|A+B| \kappa(4 / 3)^{d},
\end{aligned}
$$

because Corollary 3 says that $c-b$ cannot be in too many balls of the form $B_{a}\left(\left\|N_{a}-a\right\|\right)$. On the other hand, let $\Upsilon_{b}$ be the set of $a \in A$ for which

$$
\left|(A+B) \cap B_{a+b}\left(\left\|N_{a}-a\right\|\right)\right|>\frac{2 \kappa}{\epsilon}(4 / 3)^{d} \frac{|A+B|}{|A|} .
$$

Comparing with (5) we have

$$
\left|\Upsilon_{b}\right| \frac{2 \kappa}{\epsilon}(4 / 3)^{d} \frac{|A+B|}{|A|}<\sum_{a \in \Upsilon_{b}}\left|(A+B) \cap B_{a+b}\left(\left\|N_{a}-a\right\|\right)\right| \leq|A+B| \kappa(4 / 3)^{d}
$$

and hence $\left|\Upsilon_{b}\right|<\frac{\epsilon}{2}|A|$.

Define

$$
A_{c}:=\left\{a \in A: c-a=b \text { for some } b \in B \text {, and } a \notin \Upsilon_{b}\right\}
$$


and $N_{c}^{(l)}$ be the set of $l$ nearest neighbours to $c$ in $A+B$, where $l=1+\left[\frac{2 \kappa}{\epsilon}(4 / 3)^{d}|A+B| /|A|\right]$. If $a \in A_{c}$ and $c=a+b$ then $a \notin \Upsilon_{b}$ so, $B_{c}\left(\left\|N_{a}-a\right\|\right)$ contains $<l$ elements of $A+B$, though it does contain $N_{a}+b$, and therefore $N_{a}+b \in N_{c}^{(l)}$. Hence

$$
N_{a}-a=\left(N_{a}+b\right)-c \in N_{c}^{(l)}-c .
$$

This means that there are $\leq l$ choices for $N_{a}-a$, as $a$ runs over all of the elements of $A_{c}$.

Let $R_{1}=\emptyset$. Select $c_{1} \in A+B$ so that $A_{c_{1}} \backslash R_{1}$ is of maximal size, and then let $R_{2}=A_{c_{1}} \cup R_{1}$. More generally, given $R_{k}$ we select $c_{k} \in A+B$ so that $A_{c_{k}} \backslash R_{k}$ is of maximal size, and then let $R_{k+1}=A_{c_{k}} \cup R_{k}=\bigcup_{j=1}^{k} A_{c_{j}}$, for each $k \geq 1$. Therefore

$$
\begin{aligned}
\sum_{c \in A+B}\left|A_{c} \backslash R_{k}\right| & =\sum_{b \in B} \#\left\{a \in A \backslash R_{k}: a \notin \Upsilon_{b}\right\} \\
& \geq \sum_{b \in B}\left(|A|-\left|R_{k}\right|-\left|\Upsilon_{b}\right|\right) \\
& \geq|B|\left(\left(1-\frac{\epsilon}{2}\right)|A|-\left|R_{k}\right|\right) .
\end{aligned}
$$

Hence, by definition, $A_{c_{k}}$ satisfies

$$
\left|A_{c_{k}} \backslash R_{k}\right| \geq \frac{1}{|A+B|} \sum_{c \in A+B}\left|A_{c} \backslash R_{k}\right| \geq \frac{|A||B|}{|A+B|}\left(1-\frac{\epsilon}{2}-\frac{\left|R_{k}\right|}{|A|}\right) .
$$

We define $\theta_{j} \in[0,1]$ by $\left|R_{j}\right|=\left(1-\theta_{j}\right)|A|$, so that $\left(\theta_{j}\right)_{j \geq 1}$ is a decreasing sequence. By (4) we have

$$
\left(\theta_{k}-\theta_{k+1}\right)|A|=\left|R_{k+1}\right|-\left|R_{k}\right|=\left|A_{c_{k}} \backslash R_{k}\right| \geq \frac{|A||B|}{|A+B|}\left(\theta_{k}-\frac{\epsilon}{2}\right),
$$

Therefore if $\theta_{k} \geq \epsilon$ then

$$
\theta_{k+1} \leq\left(1-\frac{|B|}{2|A+B|}\right) \theta_{k}
$$

We deduce that $\theta_{n}<\epsilon$ for some $n \ll \frac{|A+B|}{|B|} \log (1 / \epsilon)$, else $\theta_{k} \geq \epsilon$ for all $k \leq n$ and so

$$
\theta_{n} \leq\left(1-\frac{|B|}{2|A+B|}\right)^{n-1}<\epsilon
$$

as $\theta_{1}=1$.

Finally we let $A^{\prime}=R_{n}$ so that $A^{\prime} \subset A$ with $\left|A^{\prime}\right|>(1-\epsilon)|A|$. Now $A^{\prime}$ is the union of $n-1$ sets of the form $A_{c_{j}}$; for each $j$ we select $a_{j} \in A_{c_{j}}$ which maximizes $\mid(A+B) \cap$ $B_{c_{j}}\left(\left\|N_{a_{j}}-a_{j}\right\|\right) \mid$. Therefore

$$
\left|\left\{N_{a}-a: a \in A^{\prime}\right\}\right| \leq \sum_{j=1}^{n-1}\left|(A+B) \cap B_{c_{j}}\left(\left\|N_{a_{j}}-a_{j}\right\|\right)\right|<n l \ll(4 / 3)^{d} \cdot \frac{|A+B|^{2}}{|A||B|} \cdot \frac{1}{\epsilon} \log \frac{1}{\epsilon},
$$

and hence the result. 
Example. We construct an example

$$
A=\left\{1,4,9,16, \ldots, m^{2}, m^{2}+1, m^{2}+2, \ldots, 2 m^{2}-m\right\} \subset \mathbb{R}
$$

and convert the example to $\mathbb{R} / \mathbb{Z}$, by considering $\left\{a / 4 m^{2}: a \in A\right\} \subset \mathbb{R} / \mathbb{Z}$. Now $|A|=$ $m^{2},|A+A|<4 m^{2}$ and $\left|\left\{N_{a}-a: a \in A\right\}\right| \geq m$. So if we take $\epsilon=1 /(2 m)$ then, for any subset $A^{\prime} \subset A$ with $\left|A^{\prime}\right|>(1-\epsilon)|A|$. we have

$$
\left|\left\{N_{a}-a: a \in A^{\prime}\right\}\right| \geq\left|\left\{N_{a}-a: a \in A\right\}\right|-\left|A \backslash A^{\prime}\right| \geq m-\epsilon m^{2} \geq m / 2
$$

whereas

$$
(4 / 3)^{d} \cdot \min _{B} \frac{|A+B|^{2}}{|A||B|} \cdot \frac{1}{\epsilon} \log \frac{1}{\epsilon} \leq(4 / 3) \frac{|A+A|^{2}}{|A|^{2}} \cdot 2 m \log 2 m \ll m \log 2 m .
$$

This exhibits that the upper bound in Theorem 4 cannot be much improved.

Acknowledgements: Thanks to Henry Cohn for some help with sphere packing, and Boris Bukh and Seva Lev for their remarks.

\section{REFERENCES}

1. F. A. Behrend, On sets of integers which contain no three terms in arithmetic progression, Proc. Nat. Acad. Sci. 32 (1946), 331-332.

2. F.R.K. Chung and R.L. Graham, On the set of distances determined by the union of arithmetic progressions, Ars Combinatoria 1 (1976), 57-76.

3. C. Cobeli, G. Groza, M. Vâjâitu and A. Zaharescu, Generalization of a theorem of Steinhaus, Colloq. Math 92 (2002), 257-266.

4. J.H. Conway and N.J.A. Sloane, Sphere packings, lattices and groups., Springer-Verlag, New York, 1999, pp. 703.

5. G. Groza, M. Vâjâitu and A. Zaharescu, Primitive arcs on elliptic curves, Rev. Roumaine Math. Pures Appl 50 (2005), 31-38.

6. F.M. Liang, A short proof of the 3d distance theorem, Discrete Math 28 (1979), 325-326.

7. V.T. Sós, On the theory of diophantine approximations, Acta Math. Acad. Sci. Hungar 8 (1957), 461-472.

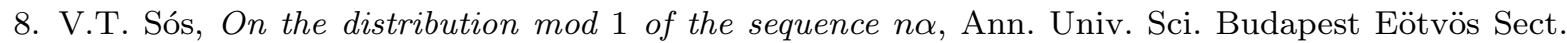
Math 1 (1958), 127-134.

9. S. Swierczkowski, On successive settings of an arc on the circumference of a circle, Fund. Math. 46 (1958), 187-189.

10. M. Vâjâitu and A. Zaharescu, Distinct gaps between fractional parts of sequences, Proc. Amer. Math. Soc 130 (2002), 3447-3452. 